\title{
Partial Dynamical Symmetry in a Fermion System
}

\author{
Jutta Eschert and Amiram Leviatan** \\ Racah Institute of Physics, The Hebrew University, Jerusalem 91904, Israel
}

(October 25, 2018)

\begin{abstract}
The relevance of the partial dynamical symmetry concept for an interacting fermion system is demonstrated. Hamiltonians with partial SU(3) symmetry are presented in the framework of the symplectic shell-model of nuclei and shown to be closely related to the quadrupole-quadrupole interaction. Implications are discussed for the deformed light nucleus ${ }^{20} \mathrm{Ne}$.
\end{abstract}

PACS numbers: 21.60Fw, 21.10-k, 21.60.Cs, 27.30+t

Symmetries play an important role in dynamical systems. They provide labels for the classification of states, determine selection rules, and simplify the relevant Hamiltonian matrices. Algebraic, symmetry-based models offer significant simplifications when the Hamiltonian under consideration commutes with all the generators of a particular group ('exact symmetry') or when it is written in terms of the Casimir operators of a chain of nested groups ('dynamical symmetry') [1]. In both cases basis states belonging to inequivalent irreducible representations (irreps) of the relevant groups do not mix, the Hamiltonian matrix has block structure, and all properties of the system can be expressed in closed form. An exact or dynamical symmetry not only facilitates the numerical treatment of the Hamiltonian, but also its interpretation and thus provides considerable insight into the physics of a given system.

Naturally, the application of exact or dynamical symmetries to realistic situations has its limitations. Usually the assumed symmetry is only approximately fulfilled, and imposing certain symmetry requirements on the Hamiltonian might result in constraints which are too severe and incompatible with experimentally observed features of the system. The standard approach in such situations is to break the symmetry. Partial Dynamical Symmetry (PDS) [2] corresponds to a particular symmetry-breaking for which the Hamiltonian is not invariant under the symmetry group and hence various irreps are mixed in its eigenstates, yet it possess a subset of 'special' solvable states which respect the symmetry. This new scheme has recently been introduced in bosonic systems and has been applied to the spectroscopy of deformed nuclei [3] and to the study of mixed systems with coexisting regularity and chaos [4]. It is the purpose of this Letter to demonstrate the relevance of the partial dynamical symmetry concept to fermion systems. More specifically, in the framework of the symplectic shellmodel of nuclei [5], we will prove the existence of a family of fermionic Hamiltonians with partial SU(3) symmetry. The PDS Hamiltonians are rotationally invariant and closely related to the quadrupole-quadrupole interaction; hence our study will shed new light on this important interaction. We will compare the spectra and eigenstates of the quadrupole-quadrupole and PDS Hamiltonians for the deformed light nucleus ${ }^{20} \mathrm{Ne}$.
The quadrupole-quadrupole interaction is an important ingredient in models that aim at reproducing quadrupole collective properties of nuclei. A model which is able to fully accommodate the action of the collective quadrupole operator, $Q_{2 m}=\sqrt{\frac{16 \pi}{5}} \sum_{s} r_{s}^{2} Y_{2 m}\left(\hat{r}_{s}\right)$, is the symplectic shell model (SSM), an algebraic scheme which respects the Pauli exclusion principle [5]. In the SSM, this operator takes the form $Q_{2 m}=\sqrt{3}\left(\hat{C}_{2 m}^{(11)}+\hat{A}_{2 m}^{(20)}+\right.$ $\hat{B}_{2 m}^{(02)}$ ), where $\hat{A}_{l m}^{(20)}, \hat{B}_{l m}^{(02)}$, and $\hat{C}_{l m}^{(11)}$ are symplectic generators with good $\mathrm{SU}(3)$ [superscript $(\lambda, \mu)$ ] and $\mathrm{SO}(3)$ [subscript $l, m$ ] tensorial properties. The $\hat{A}_{l m}^{(20)}\left(\hat{B}_{l m}^{(02)}\right), l$ $=0$ or 2 , create (annihilate) $2 \hbar \omega$ excitations in the system. The $\hat{C}_{l m}^{(11)}, l=1$ or 2 , generate a $\mathrm{SU}(3)$ subgroup and act only within one harmonic oscillator (h.o.) shell $\left(\sqrt{3} \hat{C}_{2 m}^{(11)}=Q_{2 m}^{E}\right.$, the symmetrized quadrupole operator of Elliott, which does not couple different h.o. shells [6], and $\hat{C}_{1 m}^{(11)}=\hat{L}_{m}$, the orbital angular momentum operator). A fermion realization of these generators is given in [7].

A basis for the symplectic model is generated by applying symmetrically coupled products of the $2 \hbar \omega$ raising operator $\hat{A}^{(20)}$ with itself to the usual $0 \hbar \omega$ many-particle shell-model states. Each $0 \hbar \omega$ starting configuration is characterized by the distribution of oscillator quanta into the three cartesian directions, $\left\{\sigma_{1}, \sigma_{2}, \sigma_{3}\right\}\left(\sigma_{1} \geq \sigma_{2} \geq\right.$ $\left.\sigma_{3}\right)$, or, equivalently, by its $\mathrm{U}(1) \times \mathrm{SU}(3)$ quantum numbers $N_{\sigma}\left(\lambda_{\sigma}, \mu_{\sigma}\right)$. Here $\lambda_{\sigma}=\sigma_{1}-\sigma_{2}, \mu_{\sigma}=\sigma_{2}-\sigma_{3}$ are the Elliott $\mathrm{SU}(3)$ labels, and $N_{\sigma}=\sigma_{1}+\sigma_{2}+\sigma_{3}$ is related to the eigenvalue of the oscillator number operator. The product of $N / 2$ raising operators $\hat{A}^{(20)}$ generates $N \hbar \omega$ excitations for each starting irrep $N_{\sigma}\left(\lambda_{\sigma}, \mu_{\sigma}\right)$. Each such product operator $\mathcal{P}^{N\left(\lambda_{n}, \mu_{n}\right)}$, labeled according to its $\mathrm{SU}(3)$ content, $\left(\lambda_{n}, \mu_{n}\right)$, is coupled with $\left|N_{\sigma}\left(\lambda_{\sigma}, \mu_{\sigma}\right)\right\rangle$ to good SU(3) symmetry $\rho(\lambda, \mu)$, with $\rho$ denoting the multiplicity of the coupling $\left(\lambda_{n}, \mu_{n}\right) \otimes\left(\lambda_{\sigma}, \mu_{\sigma}\right)$. The quanta distribution in the resulting state is given by $\left\{\omega_{1}, \omega_{2}, \omega_{3}\right\}$, with $N_{\sigma}+N=\omega_{1}+\omega_{2}+\omega_{3}, \omega_{1} \geq \omega_{2} \geq \omega_{3}$, and $\lambda=\omega_{1}-\omega_{2}, \mu=\omega_{2}-\omega_{3}$. The basis state construction is schematically illustrated in Fig. 1 for a typical Elliott starting state with $\left(\lambda_{\sigma}, \mu_{\sigma}\right)=(\lambda, 0) .{ }^{20} \mathrm{Ne}$, for instance, has $N_{\sigma}=48.5$ (after removal of the center-ofmass contribution) and $\left(\lambda_{\sigma}, \mu_{\sigma}\right)=(8,0)$ [5,8]. To complete the basis state labeling, additional quantum num- 


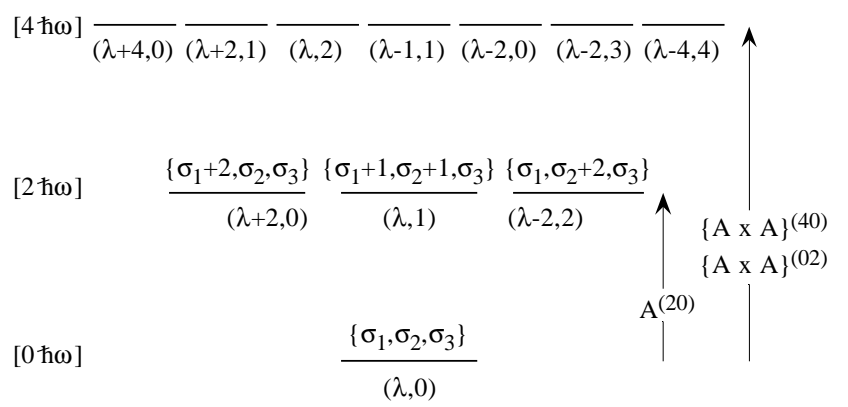

FIG. 1. Basis construction in the symplectic model. $\mathrm{SU}(3)$-coupled products of the raising operator $\hat{A}^{(20)}$ with itself act on an Elliott starting state with $\left(\lambda_{\sigma}, \mu_{\sigma}\right)=(\lambda, 0)$ $\left(\left\{\sigma_{1}, \sigma_{2}, \sigma_{3}=\sigma_{2}\right\}\right)$ to generate symplectic $2 \hbar \omega, 4 \hbar \omega, \ldots$ excitations. Also shown are the $\mathrm{SU}(3)$ labels $(\lambda, \mu)$ and quanta distributions $\left\{\omega_{1}, \omega_{2}, \omega_{3}\right\}$ for some excited states.

bers $\alpha=\kappa L M$ are required, where $L$ denotes the angular momentum with projection $M$, and $\kappa$ is a multiplicity index, which enumerates multiple occurrences of a particular $L$ value in the $\mathrm{SU}(3)$ irrep $(\lambda, \mu)$ from 1 to $\kappa_{L}^{\max }(\lambda, \mu)=[(\lambda+\mu+2-L) / 2]-[(\lambda+1-L) / 2]-$ $[(\mu+1-L) / 2]$, where [...] is the greatest non-negative integer function [9]. The group chain corresponding to this labeling scheme is $\mathrm{Sp}(6, \mathrm{R}) \supset \mathrm{SU}(3) \supset \mathrm{SO}(3)$ which defines a dynamical symmetry basis.

The quadrupole-quadrupole interaction connects h.o. states differing in energy by $0 \hbar \omega, \pm 2 \hbar \omega$, and $\pm 4 \hbar \omega$, and may be written as

$$
\begin{aligned}
Q_{2} \cdot Q_{2}= & 9 \hat{C}_{S U 3}-3 \hat{C}_{S p 6}+\hat{H}_{0}^{2}-2 \hat{H}_{0}-3 \hat{L}^{2}-6 \hat{A}_{0} \hat{B}_{0} \\
& +\{\text { terms coupling different h.o. shells }\}, \quad(1)
\end{aligned}
$$

where $\hat{C}_{S U 3}$ and $\hat{C}_{S p 6}$ are the quadratic Casimir invariants of SU(3) and $\mathrm{Sp}(6, \mathrm{R})$ with eigenvalues $2\left(\lambda^{2}+\mu^{2}+\right.$ $\lambda \mu+3 \lambda+3 \mu) / 3$ and $2\left(\lambda_{\sigma}^{2}+\mu_{\sigma}^{2}+\lambda_{\sigma} \mu_{\sigma}+3 \lambda_{\sigma}+3 \mu_{\sigma}\right) / 3+$ $N_{\sigma}^{2} / 3-4 N_{\sigma}$, respectively. These operators, as well as the h.o. $\hat{H}_{0}$ and $\hat{L}^{2}$ terms, are diagonal in the dynamical symmetry basis. Unlike the Elliott quadrupole-quadrupole interaction, $Q_{2}^{E} \cdot Q_{2}^{E}=6 \hat{C}_{S U 3}-3 \hat{L}^{2}$, the $Q_{2} \cdot Q_{2}$ interaction of Eq. (11) breaks SU(3) symmetry within each h.o. shell since the term $\hat{A}_{0} \hat{B}_{0} \equiv \hat{A}_{0}^{(20)} \hat{B}_{0}^{(02)}=\left(\{\hat{A} \times \hat{B}\}_{0}^{(00)}-\right.$ $\left.\sqrt{5}\{\hat{A} \times \hat{B}\}_{0}^{(22)}\right) / \sqrt{6}$ mixes different $\mathrm{SU}(3)$ irreps. In order to study the action of $Q_{2} \cdot Q_{2}$ within such a shell, we consider the following family of Hamiltonians:

$$
\begin{aligned}
& H\left(\beta_{0}, \beta_{2}\right)=\beta_{0} \hat{A}_{0} \hat{B}_{0}+\beta_{2} \hat{A}_{2} \cdot \hat{B}_{2} \\
& =\frac{\beta_{2}}{18}\left(9 \hat{C}_{S U 3}-9 \hat{C}_{S p 6}+3 \hat{H}_{0}^{2}-36 \hat{H}_{0}\right)+\left(\beta_{0}-\beta_{2}\right) \hat{A}_{0} \hat{B}_{0} .
\end{aligned}
$$

For $\beta_{0}=\beta_{2}$, one recovers the dynamical symmetry, and with the special choice $\beta_{0}=12, \beta_{2}=18$, one obtains $Q_{2} \cdot Q_{2}=H\left(\beta_{0}=12, \beta_{2}=18\right)+\operatorname{const}(N)-3 \hat{L}^{2}+$ terms coupling different shells, where const $(N)$ is constant for a given h.o. $N \hbar \omega$ excitation.

From Eq. (2) it follows that $H\left(\beta_{0}, \beta_{2}\right)$ is not $\mathrm{SU}(3)$ invariant. We will now show that $H\left(\beta_{0}, \beta_{2}\right)$ exhibits partial
$\mathrm{SU}(3)$ symmetry. Specifically, we claim that among the eigenstates of $H\left(\beta_{0}, \beta_{2}\right)$, there exists a subset of solvable pure- $\mathrm{SU}(3)$ states, the $\mathrm{SU}(3) \supset \mathrm{SO}(3)$ classification of which depends on both the Elliott labels $\left(\lambda_{\sigma}, \mu_{\sigma}\right)$ of the starting state and the symplectic excitation $N$. In general, we find that all L-states in the starting configuration $(N=0)$ are solvable with good $\mathrm{SU}(3)$ symmetry $\left(\lambda_{\sigma}, \mu_{\sigma}\right)$. For excited configurations $(N>0$ and even $)$ we distinguish between two possible cases:

(a) $\lambda_{\sigma}>\mu_{\sigma}$ : the pure states belong to $(\lambda, \mu)=$ $\left(\lambda_{\sigma}-N, \mu_{\sigma}+N\right)$ and have $L=\mu_{\sigma}+N, \mu_{\sigma}+$ $N+1, \ldots, \lambda_{\sigma}-N+1$ with $N=2,4, \ldots$ subject to $2 N \leq\left(\lambda_{\sigma}-\mu_{\sigma}+1\right)$.

(b) $\lambda_{\sigma} \leq \mu_{\sigma}$ : the special states belong to $(\lambda, \mu)=\left(\lambda_{\sigma}+\right.$ $\left.N, \mu_{\sigma}\right)$ and have $L=\lambda_{\sigma}+N, \lambda_{\sigma}+N+1, \ldots, \lambda_{\sigma}+$ $N+\mu_{\sigma}$ with $N=2,4, \ldots$

To prove the claim, it suffices to show that $\hat{B}_{0}$ annihilates the states in question. For $N=0$ this follows immediately from the fact that the $0 \hbar \omega$ starting configuration is a $\operatorname{Sp}(6, \mathrm{R})$ lowest weight which, by definition, is annihilated by the lowering operators of the $\operatorname{Sp}(6, \mathrm{R})$ algebra. The latter include the generators $\hat{B}_{l m}^{(02)}$. For $N>0$, let $\left\{\sigma_{1}, \sigma_{2}, \sigma_{3}\right\}$ be the quanta distribution for a $0 \hbar \omega$ state with $\lambda_{\sigma}>\mu_{\sigma}$. Adding $N$ quanta to the 2direction yields a $N \hbar \omega$ state with quanta distribution $\left\{\sigma_{1}, \sigma_{2}+N, \sigma_{3}\right\}$, that is $(\lambda, \mu)=\left(\lambda_{\sigma}-N, \mu_{\sigma}+N\right)$. Acting with the rotational invariant $\hat{B}_{0}$ on such a state does not affect the angular momentum, but removes two quanta from the 2-direction, giving a $(N-2) \hbar \omega$ state with $\left(\lambda^{\prime}, \mu^{\prime}\right)=\left(\lambda_{\sigma}-N+2, \mu_{\sigma}+N-2\right)$. (The symplectic generator $\hat{B}_{0}$ cannot remove quanta from the other two directions of this particular state, since this would yield a state belonging to a different symplectic irrep.) Comparing the number of $L$ occurrences in $(\lambda, \mu)$ and $\left(\lambda^{\prime}, \mu^{\prime}\right)$, one finds that as long as $\lambda_{\sigma}-N+$ $1 \geq \mu_{\sigma}+N, \Delta_{L}(N) \equiv \kappa_{L}^{\max }(\lambda, \mu)-\kappa_{L}^{\max }\left(\lambda^{\prime}, \mu^{\prime}\right)=1$ for $L=\mu_{\sigma}+N, \mu_{\sigma}+N+1, \ldots, \lambda_{\sigma}-N+1$, and $\Delta_{L}(N)=0$ otherwise. When $\Delta_{L}(N)=1$, a linear combination $\left|\phi_{L}(N)\right\rangle=\sum_{\kappa} c_{\kappa}\left|N \hbar \omega\left(\lambda_{\sigma}-N, \mu_{\sigma}+N\right) \kappa L M\right\rangle$ exists such that $\hat{B}_{0}\left|\phi_{L}(N)\right\rangle=0$, and thus our claim for family (a) holds. The proof for family (b) can be carried out analogously if one considers adding $N$ quanta to the 1-direction of the starting irrep. In this case there is no restriction on $\mathrm{N}$, hence family (b) is infinite.

The special states have well defined symmetry $\operatorname{Sp}(6, \mathrm{R})$ $\supset \mathrm{SU}(3) \supset \mathrm{SO}(3)$ and are annihilated by $\hat{B}_{0}$. This ensures that they are solvable eigenstates of $H\left(\beta_{0}, \beta_{2}\right)$ with eigenvalues $E(N=0)=0, E(N)=\beta_{2} N\left(N_{\sigma}-\right.$ $\left.\lambda_{\sigma}+\mu_{\sigma}-6+3 N / 2\right) / 3$ for family (a), and $E(N)=$ $\beta_{2} N\left(N_{\sigma}+2 \lambda_{\sigma}+\mu_{\sigma}-3+3 N / 2\right) / 3$ for family (b). All $0 \hbar \omega$ states are unmixed and span the entire $\left(\lambda_{\sigma}, \mu_{\sigma}\right)$ irrep. In contrast, for the excited levels $(N>0)$, the pure states span only part of the corresponding $\mathrm{SU}(3)$ irreps. There are other states at each excited level which do not preserve the SU(3) symmetry and therefore con- 


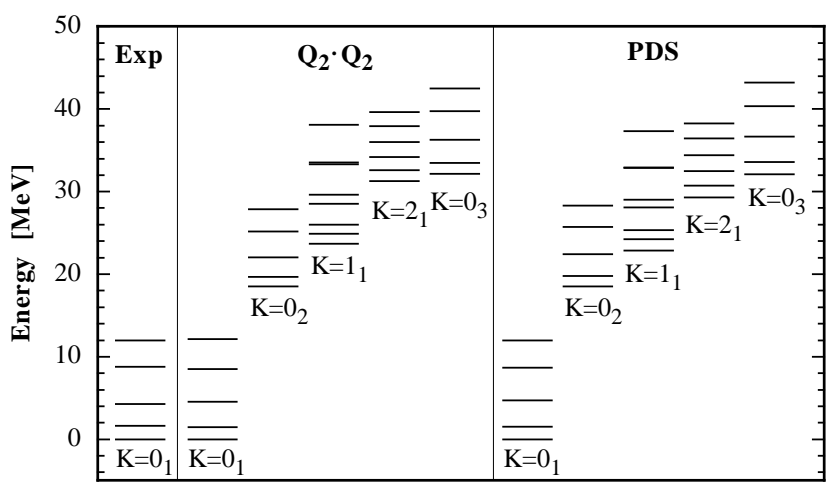

FIG. 2. Energy spectra for ${ }^{20} \mathrm{Ne}$. Comparison between experimental values (left), results from a symplectic $8 \hbar \omega$ calculation (center) and a PDS calculation (right). The angular momenta of the positive parity states in the rotational bands are $L=0,2,4, \ldots$ for $\mathrm{K}=0$ and $L=\mathrm{K}, \mathrm{K}+1, \mathrm{~K}+2, \ldots$ otherwise.

tain a mixture of $\mathrm{SU}(3)$ irreps. The partial $\mathrm{SU}(3)$ symmetry of $H\left(\beta_{0}, \beta_{2}\right)$ is converted into partial dynamical $\mathrm{SU}(3)$ symmetry by adding to it $\mathrm{SO}(3)$ rotation terms which lead to $\mathrm{L}(\mathrm{L}+1)$-type splitting but do not affect the wave functions. The solvable states then form rotational bands and since their wave functions are known, one can evaluate the E2 rates between them [10]. It is of interest to note that both the fermion Hamiltonian presented here and the boson Hamiltonian of [3] exhibit partial $\mathrm{SU}(3)$ symmetry and involve a $\mathrm{SU}(3)$ tensor of the form $[(2,0) \times(0,2)](2,2) L=0$.

To illustrate that the PDS Hamiltonians of Eq. (2) are physically relevant, we compare the eigenstates of $H_{P D S}=h(N)+\xi H\left(\beta_{0}=12, \beta_{2}=18\right)+\gamma_{2} \hat{L}^{2}+\gamma_{4} \hat{L}^{4}$ to those of the symplectic Hamiltonian $H_{S p 6}=\hat{H}_{0}-\chi Q_{2}$. $Q_{2}+d_{2} \hat{L}^{2}+d_{4} \hat{L}^{4}$. Here the function $h(N)$ is simply a constant for a given $N \hbar \omega$ excitation and contains the h.o. term $\hat{H}_{0}$. Least squares fits to measured energies and $\mathrm{B}(\mathrm{E} 2)$ values of the ground band of ${ }^{20} \mathrm{Ne}$ were carried out for $2 \hbar \omega, 4 \hbar \omega, 6 \hbar \omega$, and $8 \hbar \omega$ symplectic model spaces. The resulting energies and transition rates converge to values which agree with the data, Fig. 2 and Table [1. The parameters $\gamma_{2}$ and $\gamma_{4}$ in $H_{P D S}$ were determined by the energy splitting between states of the ground band, $\xi$ was adjusted to reproduce the relative

TABLE I. B(E2) values (in Weisskopf units) for ground band transitions in ${ }^{20} \mathrm{Ne}$. Compared are several symplectic calculations, PDS results, and experimental data 13. The static quadrupole moment of the $2_{1}^{+}$state is given in the last row. PDS results are rescaled by an effective charge $\mathrm{e}^{*}=1.95$ and the symplectic calculations employ bare charges.

\begin{tabular}{ccccccc}
\hline \hline Transition & \multicolumn{5}{c}{ Model B(E2) $[$ W.u.] } & B(E2) [W.u.] \\
\cline { 2 - 6 }$J_{i} \rightarrow J_{f}$ & $2 \hbar \omega$ & $4 \hbar \omega$ & $6 \hbar \omega$ & $8 \hbar \omega$ & PDS & Exp. \\
\hline $2 \rightarrow 0$ & 14.0 & 18.7 & 19.1 & 19.3 & 20.3 & $20.3 \pm 1.0$ \\
$4 \rightarrow 2$ & 18.4 & 24.5 & 24.6 & 24.5 & 25.7 & $22.0 \pm 2.0$ \\
$6 \rightarrow 4$ & 17.1 & 22.3 & 21.5 & 20.9 & 21.8 & $20.0 \pm 3.0$ \\
$8 \rightarrow 6$ & 12.4 & 15.2 & 13.3 & 12.4 & 12.9 & $9.0 \pm 1.3$ \\
\hline$Q[\mathrm{eb}]$ & -0.14 & -0.16 & -0.16 & -0.16 & -0.17 & $-0.23 \pm 0.03$ \\
\hline \hline
\end{tabular}

positions of the resonance bandheads and $h(N)$ was fixed by the energy difference $\left[E\left(0_{2}^{+}\right)-E\left(0_{1}^{+}\right)\right]$. Fig. 2 and Table demonstrate the level of agreement between the PDS and symplectic results.

An analysis of the structure of the ground and resonance bands reveals the amount of mixing in the $8 \hbar \mathrm{\omega}$ symplectic $\left(Q_{2} \cdot Q_{2}\right)$ wave functions. Fig. 3 shows the decomposition for representative $\left(2^{+}\right)$states of the five lowest rotational bands. Ground band $\left(\mathrm{K}=0_{1}\right)$ states are found to have a strong $0 \hbar \omega$ component $(\geq 64 \%)$, and three of the four resonance bands are clearly dominated $(\geq 60 \%)$ by $2 \hbar \omega$ configurations. States of the first resonance band $\left(K=0_{2}\right)$, however, contain significant contributions from all but the highest $N \hbar \omega$ excitations. The relative strengths of the $\mathrm{SU}(3)$ irreps within the $2 \hbar \omega$ space are shown as well: states are found to be dominated by one representation $\left[(10,0)\right.$ for the $\mathrm{K}=\mathrm{O}_{2}$ band, $(8,1)$ for $\mathrm{K}=1_{1},(6,2) \kappa=2$ for $\mathrm{K}=2_{1}$, and $(6,2) \kappa=1$ for $\mathrm{K}=0_{3}$, where $\kappa=1$ and 2 correspond here to Vergados basis labels 0 and 2, respectively [11]], while the other irreps contribute only a few percent. Such trends are present also in the more realistic symplectic calculations of [12].

The PDS Hamiltonian $H_{P D S}$ acts only within one oscillator shell, hence its eigenfunctions do not contain admixtures from different $N \hbar \omega$ configurations. As expected, $H_{P D S}$ has families of pure $\mathrm{SU}(3)$ eigenstates which can be organized into rotational bands. The ground band belongs entirely to $N=0,(\lambda, \mu)=(8,0)$, and all states of the $\mathrm{K}=2_{1}$ band have quantum labels $N=2,(\lambda, \mu)=(6,2), \kappa=2$. A comparison with the symplectic case shows that the $N \hbar \omega$ level to which a particular PDS band belongs is also dominant in the corresponding symplectic band. In addition, within this dominant excitation, eigenstates of $H_{P D S}$ and $H_{S p 6}$ have similar SU(3) distributions; in particular, both Hamiltonians favor the same $(\lambda, \mu) \kappa$ values. Significant differences in the structure of the wave functions appear, however, for the $\mathrm{K}=0_{2}$ resonance band. In the $8 \hbar \omega$ symplectic calculation, this band contains almost equal contributions from the $0 \hbar \omega, 2 \hbar \omega$, and $4 \hbar \omega$ levels, with additional admixtures of $6 \hbar \omega$ and $8 \hbar \omega$ configurations, while in the PDS calculation, it belongs entirely to the $2 \hbar \omega$ level. These structural differences are also evident in the interband transition rates, e.g. $\mathrm{B}\left(\mathrm{E} 2 ; \mathrm{K}=0_{1}, \mathrm{~L}=2^{+} \rightarrow \mathrm{K}=0_{2}, \mathrm{~L}=0^{+}\right)$ $=2.93$ (5.69) W.u. and $\mathrm{B}\left(\mathrm{E} 2 ; \mathrm{K}=0_{2}, \mathrm{~L}=2^{+} \rightarrow \mathrm{K}=0_{1}\right.$, $\left.\mathrm{L}=0^{+}\right)=5.84(12.6)$ W.u. in the $8 \hbar \omega(\mathrm{PDS})$ calculation, and reflect the action of the inter-shell coupling terms in Eq. (11). Increasing the strength $\chi$ of $Q_{2} \cdot Q_{2}$ in $H_{S p 6}$ will also spread the other resonance bands over many $N \hbar \omega$ excitations. The $\mathrm{K}=2_{1}$ band (which is pure in the PDS scheme) is found to resist this spreading more strongly than the other resonances. For physically relevant values of $\chi$, the low-lying bands have the structure shown in Fig. 3 .

In summary, we have introduced a family of fermionic Hamiltonians with partial SU(3) symmetry. Using the framework of the symplectic shell model, we have proven 

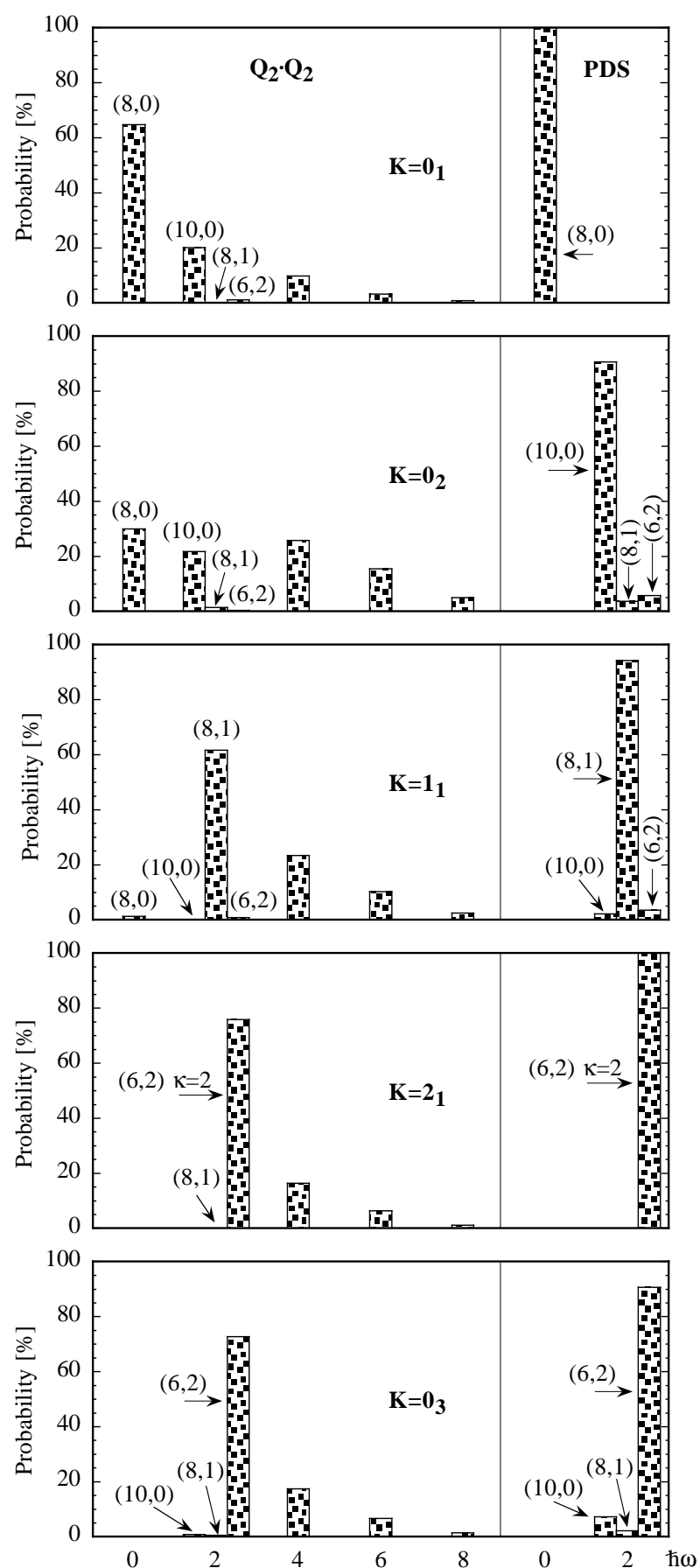

FIG. 3. Decomposition for calculated $2^{+}$states of ${ }^{20} \mathrm{Ne}$. Individual contributions from the relevant $\mathrm{SU}(3)$ irreps at the $0 \hbar \omega$ and $2 \hbar \omega$ levels are shown for both a symplectic $8 \hbar \omega$ calculation (denoted $Q_{2} \cdot Q_{2}$ ) and a PDS calculation. In addition, the total strengths contributed by the $N \hbar \omega$ excitations for $N>2$ are given for the symplectic case.

that these Hamiltonians possess both mixed-symmetry and solvable pure-SU(3) rotational bands. For the deformed light nucleus ${ }^{20} \mathrm{Ne}$, we have shown that vari- ous features of the quadrupole-quadrupole interaction can be reproduced with a particular parameterization of the PDS Hamiltonians. For both the ground and the resonance bands, PDS eigenstates were seen to approximately reproduce the structure of the exact $Q_{2} \cdot Q_{2}$ eigenstates within the $0 \hbar \omega$ and $2 \hbar \omega$ spaces, respectively. In particular, for each pure state of the PDS scheme we found a corresponding eigenstate of the quadrupolequadrupole interaction, which was dominated by the same SU(3) irrep. Moreover, for reasonable interaction parameters, each rotational band was primarily located in one level of excitation, with the exception of the lowest $\mathrm{K}=\mathrm{O}_{2}$ resonance band, which was spread over many $N \hbar \omega$ excitations. Implications of the structural differences between the various resonance bands for giant monopole and quadrupole transitions remain to be investigated. The occurrence of partial symmetries for fermions, as shown in this work, and for bosons, as presented in previous works [3], highlights their relevance to dynamical systems and motivates their further study.

The authors acknowledge valuable suggestions by D.J. Rowe and constructive comments by J.P. Elliott and G. Rosensteel. This work is supported by a grant from the Israel Science Foundation. We thank the Institute for Nuclear Theory at the University of Washington for its hospitality and the Department of Energy for partial support during the completion of this work.

* Current address: TRIUMF, 4004 Wesbrook Mall, Vancouver, B.C. V6T 2A3, Canada; email: escher@triumf.ca ** email: ami@vms.huji.ac.il

[1] Dynamical Groups and Spectrum Generating Algebras, eds. A. Bohm, Y. Néeman, and A. O. Barut, World Scientific, 1988.

[2] A. Leviatan, J. N. Ginocchio and M. W. Kirson, Phys. Rev. Lett. 65, 2854 (1990); Y. Alhassid and A. Leviatan, J. Phys. A 25, L1265 (1992); A. Leviatan, in Symmetry in Science VII, Plenum Press, New York, p. 383 (1994).

[3] A. Leviatan, Phys. Rev. Lett. 77, 818 (1996); A. Leviatan and I. Sinai, J. Phys. G 25, 791 (1999).

[4] N. D. Whelan, Y. Alhassid and A. Leviatan, Phys. Rev. Lett. 71, 2208 (1993); A. Leviatan and N. D. Whelan, Phys. Rev. Lett. 77, 5202 (1996).

[5] G. Rosensteel and D. J. Rowe, Phys. Rev. Lett. 38, 10 (1977); Ann. Phys. 126, 343 (1980); D. J. Rowe, Rep. Prog. Phys. 48, 1419 (1985).

[6] J. P. Elliott, Proc. Roy. Soc. A 245, 128 (1958); 245, 562 (1958).

[7] J. Escher and J. P. Draayer, J. Math. Phys. 39, 5123 (1998).

[8] J. P. Draayer, K. J. Weeks, and G. Rosensteel, Nucl. Phys. A 413, 215 (1984).

[9] R. Lopez, P. O. Hess, P. Rochford, and J. P. Draayer, J. Phys. A 23, L229 (1990).

[10] J. Escher and A. Leviatan, in preparation.

[11] J.D. Vergados, Nucl. Phys. A 111, 681 (1968).

[12] Y. Suzuki, Nucl. Phys. A 470, 119 (1987).

[13] D.R. Tilley et al. Nucl. Phys, A 636, 249 (1998). 